

Facultad de Ciencias Económicas

Universidad Nacional de La Plata

\author{
Tesis De MAestría
}

\title{
SWTRI: \\ Midiendo Protección Comercial junto a Impacto Distributivo
}

\author{
Juan Sebastián Fernández
}

Director:

Guido G. Porto

20 de mayo de 2016 


\title{
SWTRI: \\ Midiendo protección comercial junto a Impacto Distributivo
}

\author{
Juan Sebastián Fernández* \\ Director: Guido G. Porto
}

20 de mayo de 2016

\begin{abstract}
Resumen
Este trabajo se centra en el desarrollo de un índice de protección comercial que tome en cuenta el impacto distributivo generado por la política arancelaria. Para ello se parte del TRI, Trade Restrictiveness Index, o Índice de Restricción Comercial. Este índice tiene en cuenta solo los efectos de la política comercial sobre el ingreso real promedio a través de las distorsiones generadas por los aranceles. Reformulando el índice anterior mediante el empleo de la función de bienestar social Atkison se llega al SWTRI: Social Welfare Trade Restrictiveness Index, o Índice de Restricción Comercial en base al Bienestar Social. Se muestra su fórmula explícita y se explica su funcionamiento. Se encuentra que funciona como un promedio de los aranceles ponderado por el efecto sobre el bienestar social de cada uno. Implementando el índice sobre Burkina Faso se encuentra que la política comercial del país es pro-pobre, y que la redistribución se canaliza protegiendo de manera máxima a los cultivos producidos por los hogares más carenciados.
\end{abstract}

Clasificación JEL: F13, I30

Palabras clave: comercio internacional, política comercial, impacto distributivo.

\footnotetext{
*Quiero agradecer especialmente a mi director, Guido Porto, quien estuvo siempre dispuesto ayudarme en las vicisitudes inherentes al proceso del desarrollo de la tesis. Agradezco también a la Facultad de Ciencias Económicas y a la Universidad Nacional de La Plata, quienes me becaron tanto durante el transcurso de mis cursos en la maestría como para la realización de este proyecto. Finalmente, agradezco a los que estuvieron presentes en el Seminario de Avance de Tesis por sus comentarios. Naturalmente, los errores y omisiones de este trabajo son responsabilidad mía.
} 


\section{Índice}

1 Introducción 4

2 Planteo teórico 6

2.1 Fórmula explícita . . . . . . . . . . . . . . . . 6

2.2 El SWTRI como promedio ponderado $\ldots \ldots \ldots \ldots \ldots$

2.3 Desagregación en bienes . . . . . . . . . . . . . . 7

2.4 Ejemplo con dos bienes $\ldots \ldots \ldots \ldots \ldots$

3 Implementación empírica 13

3.1 Especificación del efecto de los aranceles sobre el ingreso real. . . 13

3.2 Datos de encuestas de hogares . . . . . . . . . . . . . . 15

3.3 Datos de comercio internacional . . . . . . . . . . . . . . 17

4 Resultados 19

5 Conclusiones 22

6 Bibliografía 23 


\section{Introducción}

Medir el nivel de protección comercial de un país es esencialmente un problema de agregación. Se posee una distribución de trabas comerciales sobre distintos bienes, y se busca llegar a una magnitud única representativa de la distribución. Esta magnitud única deberá discriminar a cada bien según su importancia económica. Es menester entonces determinar cuál es la "importancia" de cada bien.

El método más directo es ponderar a todos los bienes por igual, es decir, emplear un promedio simple. Una respuesta marginalmente superior es usar un promedio ponderado en cantidades importadas. Ninguno de estos métodos tiene una base concreta en la teóría económica y por ende poseen comportamientos indeseados.

Tomemos el promedio simple. Puede haber un bien en el cual un país tenga una ventaja comparativa, haciendo que el país lo exporte. Sin embargo, el arancel de ese bien puede ser no nulo. Claramente ese arancel es completamente irrelevante: la ventaja comparativa hace que el país sea exportador, no importador de ese bien. Si no existen otras trabas, el precio interno será igual al precio internacional, sin importar el nivel del arancel. El arancel podría ser 0 , 10, 50, 100\% y no tendría ningún efecto económico. Por ende, este bien debería tener ponderación cero. Sin embargo, el promedio simple lo ponderará como a cualquier otro bien importable.

El promedio ponderado también posee problemas. Podría existir un bien en el cual un país tuviese una fuerte desventaja comparativa, pero que no fuese importado por existir una traba total a su importación. Esas trabas elevarán el precio interno fuertemente, generando significativos efectos económicos. Sin embargo, como su importación es cero, para el ponderador en base a importaciones el bien es completamente irrelevante.

La literatura en la década pasada ha propuesto alternativas superadoras a estos indicadores simples. Se han desarrollado indicadores que den una respuesta concreta a qué hace que un bien sea "importante".

La respuesta dada a esta pregunta ha sido únanime: los bienes importan según su relevancia en el ingreso real de los individuos. El primer índice se denomina simplemente TRI Trade Restrictiveness Index, índice de restricción comercial (Anderson y Neary, 1992). Este índice responde a la pregunta ¿Cuál es el nivel de arancel uniforme que dejaría el ingreso real de los hogares sin cambios respecto de la actual política comercial? Por ende, una política comercial que generase fuertes variaciones del ingreso real, tendría como equivalente un nivel de arancel uniforme elevado. Dado que los cambios en el ingreso real son causados por las distorsiones creadas por la política comercial, el índice equipara importancia económica a eficiencia económica. Si bien la literatura ha enriquecido el análisis como por ejemplo incluyendo las restricciones a las exportaciones que el país sufre, siempre se ha movido bajo este mismo paradigma (Anderson y Neary, 1994, 1996, 2003, 2004; Kee, Nicita y Olearreaga, 2006) .

Sin embargo, al alterar precios relativos el comercio no solo genera efectos sobre la eficiencia sino también efectos distributivos. Por ello la visión puramente eficientista está está siendo complementada por otra que tome en cuenta estos efectos. Estos efectos pueden ser especialmente relevantes en países en desarrollo, ya que los aranceles son unos de los impuestos de más simple implementación. 
Mientras la literatura se ha dedicado a medir efectos puntuales de reformas comerciales sobre desigualdad y pobreza (Porto 2006; Fajgelbaum y Khandelwal, 2015), solo se ha movido en forma marginal hacia el análisis del efecto distributivo de la protección comercial en general. El trabajo publicado más cercano a éste es sin duda Nicita, Olarreaga y Porto (2014). Otros trabajos que se centran en este aspecto son muy recientes y aún están sin publicar (Antras, de Gortari, Itskhoki, 2015; Galle, Rodriguez-Clare, Yi 2015).

Este trabajo pretende llenar ese vacío. Tomando como base el primer trabajo de protección comercial (Anderson y Neary 1992), se vuelve a la pregunta de qué hace que un bien sea "importante". En vez de responder con eficiencia se responde con equidad. La pregunta que responderá el índice entonces no es ¿Cuál es el nivel arancelario uniforme que mantendrá el ingreso real constante? sino ¿Cuál es el nivel arancelario uniforme que mantendrá el bienestar social constante?. Así el TRI original se transforma en el SWTRI Social Welfare Trade Restrictiveness Index, índice de restricción comercial basado en bienestar social.

A partir de la reformulación de la pregunta anterior se desarrolla el índice, mostrando su determinación algebraica y graficamente. Se analiza su comportamiento ante cambios en el impacto distributivo de cada arancel y se muestra que se puede entender como un promedio de los aranceles ponderando por los efectos de la política comercial sobre el bienestar social.

Adicionalmente a modo de ejemplo se calcula el índice para Burkina Faso. Como resultado se encuentra que a medida que aumenta la preferencia por desigualdad, el SWTRI se reduce. Esto marca un sesgo pro-pobre en la política comercial del pais. Los resultados están de acuerdo a lo que obtienen Nicita, Olarreaga y Porto (2014).

El trabajo se organiza de la siguiente manera. La sección 2 presenta la resolución algebraica del indicador. Muestra que se puede entender como un promedio ponderado e identificar los efectos desagregados de cada bien para poder explicar el funcionamiento del SWTRI. En la sección 3 se explica cuál fue la metodología para implementar el SWTRI, tomando como país a Burkina Faso. En la sección 4 se presentan los resultados, calculando el SWTRI, mostrando como se comporta tanto a nivel desagregado como a distintas preferencias de desigualdad. En la sección 5 se concluye. 


\section{$2 \quad$ Planteo teórico}

\subsection{Fórmula explícita}

En esta primera sección se deriva la definición algebraica del SWTRI. El SWTRI es el nivel arancelario uniforme que mantendrá el bienestar social consante. La especificación empleada es la función de bienestar en Atkinson (1970):

$$
\begin{aligned}
& W=\frac{1}{H} \sum_{h} \frac{\left(x^{h}\right)^{1-\epsilon}}{1-\epsilon}, \epsilon \neq 1 \\
& \ln W=\frac{1}{H} \sum_{h} \ln x^{h}, \epsilon=1
\end{aligned}
$$

donde $W$ es el nivel de bienestar y $x^{h}$ es el nivel de ingreso real per cápita del hogar $h$ ( $H$ es el número total de hogares en la economía). Bajo esta especificación, el bienestar social es simplemente la suma ponderada del ingreso real familiar. Esos ponderadores están definidos por el parámetro $\epsilon$, que a su vez mide la preferencia por la igualdad del analista ${ }^{1}$. Para no duplicar el álgebra, se resuelve el caso más general de $\epsilon \neq 1$. Se llama $\tau$ al vector de aranceles que aplica el país, y $\tau_{W}$ al SWTRI. Bajo la especificación Atkinson, el SWTRI está definido implícitamente por

$$
\sum_{h}\left(x^{h}\left(\tau^{W}\right)\right)^{1-\epsilon}=\sum_{h}\left(x^{h}(\tau)\right)^{1-\epsilon}
$$

Para obtener al SWTRI de manera explícita se calcula la expansión de Taylor de $x^{h}\left(\tau^{W}\right)$ en torno a $\tau$. Se obtiene

$$
\left(x^{h}\left(\tau^{W}\right)\right)^{1-\epsilon}=\left(x^{h}(\tau)\right)^{1-\epsilon}+\sum_{i}(1-\epsilon)\left(x^{h}(\tau)\right)^{-\epsilon} \frac{\partial x^{h}}{\partial \tau_{i}}\left(\tau^{W}-\tau_{i}\right)
$$

Sumando en hogares

$$
\sum_{h}\left(x^{h}\left(\tau^{W}\right)\right)^{1-\epsilon}=\sum_{h}\left(x^{h}(\tau)\right)^{1-\epsilon}+(1-\epsilon) \sum_{h} \sum_{i}\left(x^{h}(\tau)\right)^{-\epsilon} \frac{\partial x^{h}}{\partial \tau_{i}}\left(\tau^{W}-\tau_{i}\right)
$$

Por lo que el SWTRI queda definido implícitamente por

$$
\sum_{h} \sum_{i}\left(x^{h}\left(\tau^{W}\right)\right)^{-\epsilon} \frac{\partial x^{h}}{\partial \tau_{i}}\left(\tau^{W}-\tau_{i}\right)=0
$$

Y explícitamente como

$$
\tau^{W}=\frac{\sum_{h} \sum_{i}\left(x^{h}(\tau)\right)^{-\epsilon} \frac{\partial x^{h}}{\partial \tau_{i}} \tau_{i}}{\sum_{h} \sum_{i}\left(x^{h}(\tau)\right)^{-\epsilon} \frac{\partial x^{h}}{\partial \tau_{i}}}
$$

\footnotetext{
${ }^{1}$ Equivalentemente, otros autores (Rodriguez-Clare, Yi 2015) siguen un enfoque estocástico donde los hogares están tras un "velo de la ignorancia", sin saber cuál es su posición en la distribución del ingreso. En este caso $\epsilon$ mide la aversión al riesgo.
} 


\subsection{El SWTRI como promedio ponderado}

Si bien (7) define explícitamente al SWTRI, para avanzar en su interpretación se necesita desarrollar herramientas adicionales. Para ello en esta sección se muestra que el SWTRI se puede considerar, de la misma manera que los otros índices de la literatura, como un promedio ponderado de los aranceles.

Partiendo de la ecuación (7), si se cambia el orden de las sumatorias, y se define a los ponderadores $\omega_{i}$ como

$$
\omega_{i}=\sum_{h}\left(x^{h}\right)^{-\epsilon} \frac{\partial x^{h}}{\partial \tau_{i}}
$$

Esto simplifica a $(7)$

$$
\tau_{W}=\frac{\sum_{i} \tau_{i} \omega_{i}}{\sum_{i} \omega_{i}}
$$

Para entender la mecánica del SWTRI se necesita comprender la naturaleza de $\operatorname{los} \omega_{i}$. Como se evidencia de (8) cada $\omega_{i}$ tiene dos componentes: el efecto marginal sobre el ingreso real de cada hogar de aumentar el arancel sobre el bien $i, \frac{\partial x^{h}}{\partial \tau_{i}}$ y la ponderación de cada hogar $h$ en el bienestar social $\left(x^{h}\right)^{-\epsilon}$.

El efecto marginal del arancel sobre el ingreso real del hogar $\frac{\partial x^{h}}{\partial \tau_{i}}$ tiene signo indeterminado. Por ejemplo, un hogar que sea productor del neto del bien verá su ingreso real crecer frente al aumento del precio interno inducido por el aumento del arancel, con lo el arancel poseerá un efecto marginal positivo sobre el ingreso real. Por el contrario, un hogar que es consumidor neto verá su ingreso real reducido, ya que el arancel incrementa el precio interno de los bienes que consume. Esto lleva a que el arancel tenga un efecto marginal negativo sobre este hogar.

Los ponderadores de cada hogar en el bienestar social $\left(x^{h}\right)^{-\epsilon}$, al expresarse como una función exponencial del ingreso, son siempre positivos. En este aspecto del SWTRI es que cobra relevancia $\epsilon$. Por ejemplo, si $\epsilon=0$, la función de bienestar se hace del tipo Bentham: un peso ganado por un hogar rico vale lo mismo que un peso ganado por un hogar pobre. A medida que épsilon crece, los pesos ganados por los hogares más pobres aumentan su relevancia en el bienstar general respecto de los de pesos ganados por los hogares más ricos. Con lo cual el efecto de los aranceles sobre estos hogares más pobres afectará más fuertemente al bienestar social.

El ponderador $\omega_{i}$ une a ambos elementos. Esto lo transforma en una suma de los efectos de los aranceles sobre el ingreso real de los hogares, ponderando a cada hogar en base a su posición en la distribución del ingreso. La ponderación se realiza en base a preferencias sobre igualdad. En el caso que $\epsilon=0, \omega_{i}$ es positivo (negativo) si y solo si el país es productor (consumidor) neto del bien. Pero por ejemplo si en el agregado el país es consumidor neto del bien $i, \epsilon$ es muy elevado y los hogares más pobres son productores netos del bien, aumentar los aranceles tendrá un efecto positivo sobre el ingreso real de los hogares más pobres, y como estos hogares están ponderandos fuertemente, $\omega_{i}$ será positivo.

\subsection{Desagregación en bienes}

En esta sección se derivarán las fórmulas matemáticas que expresan la variación en el bienestar social desagregadas en el aporte de cada bien. Además de ser in- 
teresante en sí mismo, este ejercicio es útil tanto para entender la naturaleza de los ponderadores dentro del SWTRI de la sección anterior como para desarrollar un marco teórico que permita comprender el funcionamiento del indicador, lo que se explicitará en la sección siguiente.

De la expansión de Taylor de $x^{h}$ alrededor de $\tau$ se puede obtener las expresión de $d W$, la variación total del bienestar generada por la unificación de todos los aranceles a un único nivel arancelario que se define $\bar{\tau}$.

$$
d W=\sum_{h} \sum_{i}\left(x^{h}\right)^{-\epsilon} \frac{\partial x^{h}}{\partial \tau_{i}}\left(\bar{\tau}-\tau_{i}\right)
$$

Como sostiene la ecuación (6) cuando $\bar{\tau}=\tau_{W}$, por definición del SWTRI la variación total del bienestar debe ser cero.

Cambiando el orden de la sumatoria y reemplazando por (8) se puede simplificar a:

$$
d W=\sum_{i} d W_{i}
$$

Definiendo a $d W_{i}$ como

$$
d W_{i}=\left(\bar{\tau}-\tau_{i}\right) \omega_{i}
$$

Con lo que el ponderador de cada bien se revela como el efecto marginal de subir el arancel en el bienestar global.

La ecuación (12) le impone ciertas propiedades matemáticas a $d W_{i}$. En primer lugar, $d W_{i}$ es lineal en $\bar{\tau}$. Esta propiedad surge de emplear una expansión de Taylor de primer orden. En segundo lugar, cuando $\bar{\tau}=\tau_{i}, d W_{i}$ es igual a cero, ya que implica que el arancel no cambia, y por ende no hay cambios en el bienestar. Como consecuencia, la abscisa al origen de $d W_{i}$ solo depende de $\tau_{i}$. Finalmente, la pendiente de $d W_{i}$ es equivalente al ponderador del bien en el SWTRI , que es a la vez el efecto marginal social de aumentar el arancel del bien. Luego si por ejemplo un país es socialmente un productor neto del bien $i$, $\omega_{i}>0$ y la pendiente de $d W_{i}$ será positiva. En cambio, si el país es socialmente un consumidor neto del bien $i, \omega_{i}<0$ y la pendiente de $d W_{i}$ será negativa.

\subsection{Ejemplo con dos bienes}

Para concluir la sección teórica se aplican todas las herramientas obtenidas en las secciones anteriores para mostrar en un ejemplo el funcionamiento del SWTRI.

Supongamos que se tienen dos bienes importables, $i=1,2$, con sus respectivos aranceles $\tau_{i}$. En la sección de desagregación se mostró que generan efectos marginales sobre el bienestar general $\omega_{i}$. Al ser bienes importables, el país es un consumidor neto de los bienes, con lo que si no tomamos en cuenta la equidad, es decir, con $\epsilon=0$ necesariamente $\omega_{1}, \omega_{2}<0$.

Definamos inicialmente $\epsilon=0$ y $\omega_{1}=\omega_{2}$. Eso implica que la participación promedio de los bienes 1 y 2 en el consumo son iguales. Pero los $\omega_{i}$, como se explicó en las sección 2.3, pueden ser interpretados como los ponderadores de los aranceles que generan a su vez el SWTRI. Como los ponderadores son iguales, el SWTRI emerge como un promedio simple. De ser las elasticidades de demanda de las importaciones de ambos bienes iguales, el TRI también sería equivalente al promedio simple. 
Si se grafica (12) bajo estos supuestos se obtiene una situación como la descripta en la Figura (1). Se ve que $d W_{i}\left(\tau_{i}\right)=0$, ya que equivale a dejar a la política comercial sin cambios. Como el SWTRI está definido implícitamente por $d W\left(\tau_{W}\right)=0$. Para obtenerlo gráficamente se debe hacer la suma de las $d W_{i}$ y ver en qué punto se intersecta con el eje de las abscisas. Ya que $d W$ es una recta solo se necesitan dos puntos para graficarla. Uno se obtiene con $d W\left(\tau_{1}\right)=d W_{2}\left(\tau_{1}\right)$, y otro con $d W\left(\tau_{2}\right)=d W_{1}\left(\tau_{2}\right)$. Uniendo ambos puntos, en el ejemplo se obtiene que $d W$ tiene pendiente negativa. Esto tiene sentido: dado que los aranceles se establecen sobre bienes que el país es consumidor neto, aumentar estos aranceles y por ende el precio interno de estos bienes reduce el bienestar general. Dado nuestros supuestos, $\tau_{W}$ se encuentra exactamente en el medio del intervalo $\left[\tau_{1}, \tau_{2}\right]$.

Figura 1: Dos bienes importables con igual $\omega_{i}$

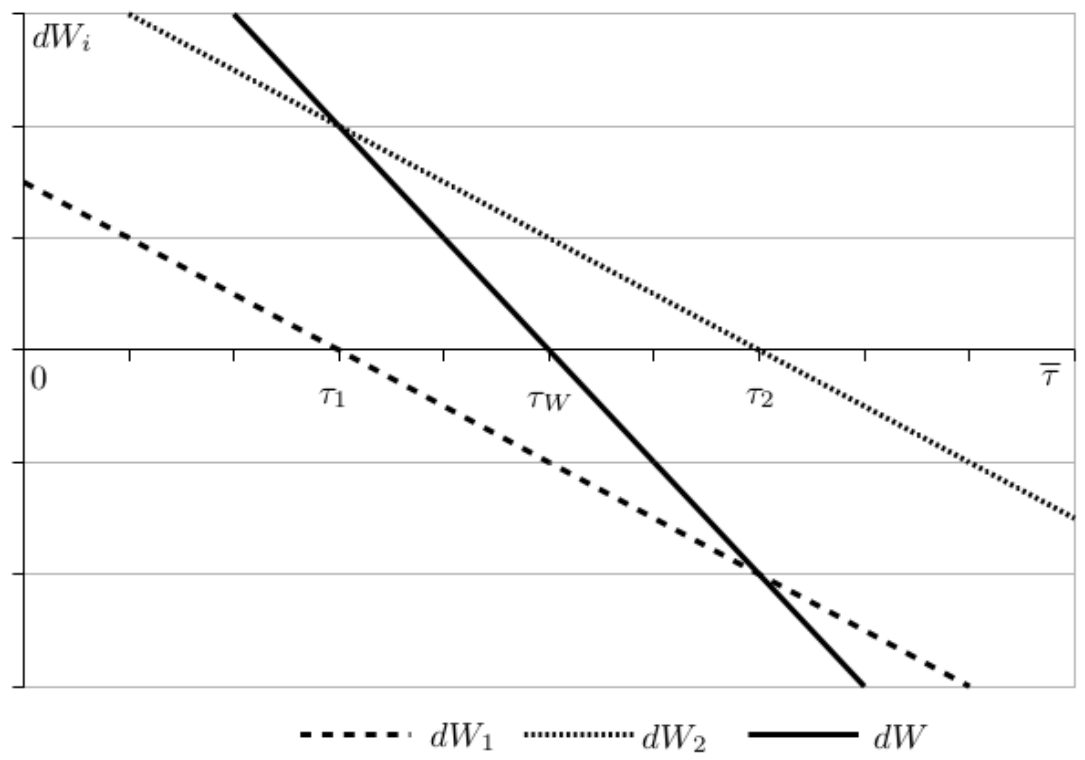

Habiendo obtenido el SWTRI se puede realizar estática comparativa para analizar su comportamiento. Supongamos que el bien 1 es consumido en iguales proporciones por hogares ricos y pobres, pero los hogares más pobres gastan una mayor parte de su ingreso en el bien 2. Si aumenta $\epsilon$, los hogares más pobres verán su ponderación en el bienestar social aumentada, y el efecto negativo de un aumento de aranceles del bien 2 también crecerá. Esto llevará a que $\omega_{2}$ se vuelva aún más negativa. Gráficamente, la pendiente de $d W_{2}$ se vuelve más empinada. En la Figura 2 se ve como esto corre el SWTRI hacia $\tau_{2}$, incrementándolo por encima del promedio simple. El país, corrigiendo por efectos sobre igualdad, se ve penalizado porque le cobra un arancel más alto al bien al cual los hogares más pobres asignan una proporción más grande de su ingreso. En el límite, con $\omega_{2}=-\infty$, el SWTRI será exactamente $\tau_{2}$. 
Figura 2: Dos bienes importables con $\omega_{2}<\omega_{1}$



¿Qué pasaría en cambio si los hogares más pobres dedican una proporción menor que los ricos de su ingreso en el bien 2? En este caso, al aumentar $\epsilon$ y por ende la ponderación de los hogares más pobres en el bienestar general, $\omega_{2}$ se reduce en valor absoluto, volviéndose $d W_{2}$ más horizontal, como muestra la Figura 3. Se ve gráficamente como el SWTRI se reduce por debajo del promedio simple, tendiendo a $\tau_{1}$. Para el SWTRI el país no es tan proteccionista, porque está usando la política comercial como herramienta redistributiva: le está cobrando aranceles más altos a los cuales los hogares más ricos asignan un porcentaje de su ingreso mayor. 
Figura 3: Dos bienes importables con $\omega_{2}<\omega_{1}$

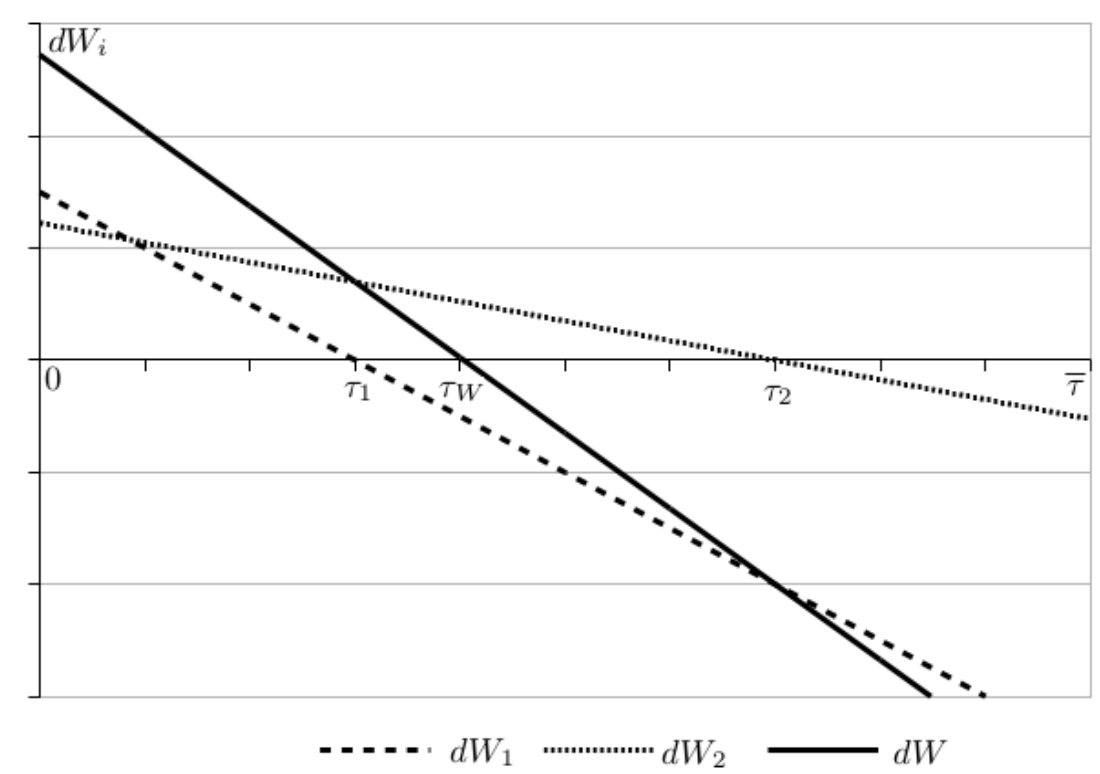

El arancel del bien 1, $\tau_{1}$ no es el límite inferior para el SWTRI. Si el bien 2 es un bien del cual los hogares más ricos son consumidores netos, los hogares más pobres productores netos, y el país en su conjunto es consumidor neto, al aumentar $\epsilon, \omega_{2}$ aumentará a tal punto de cambiar de signo. Un ejemplo de este bien podría ser algún vegetal básico producido por los hogares más pobres, quienes, sin embargo no logran abastecer todo el mercado doméstico. Como se muestra en la Figura 4, esto empuja al SWTRI por debajo de $\tau_{1}$ e incluso puede llevarlo a valores negativos. La interpretación de un SWTRI negativo es que la política comercial es tan intensamente pro-pobre gravando bajo a los bienes consumidos por los hogares de menores ingresos y alto a los bienes producidos por ellos, que para dejar el bienestar constante se debería imponer a un subsidio a todas las importaciones. Es más, al hacer tender $\omega_{2}$ a $-\omega_{1}$ el SWTRI tiende a $-\infty$. 
Figura 4: Un bien importable y otro que se vuelve exportable $\omega_{1}<0<\omega_{2}$

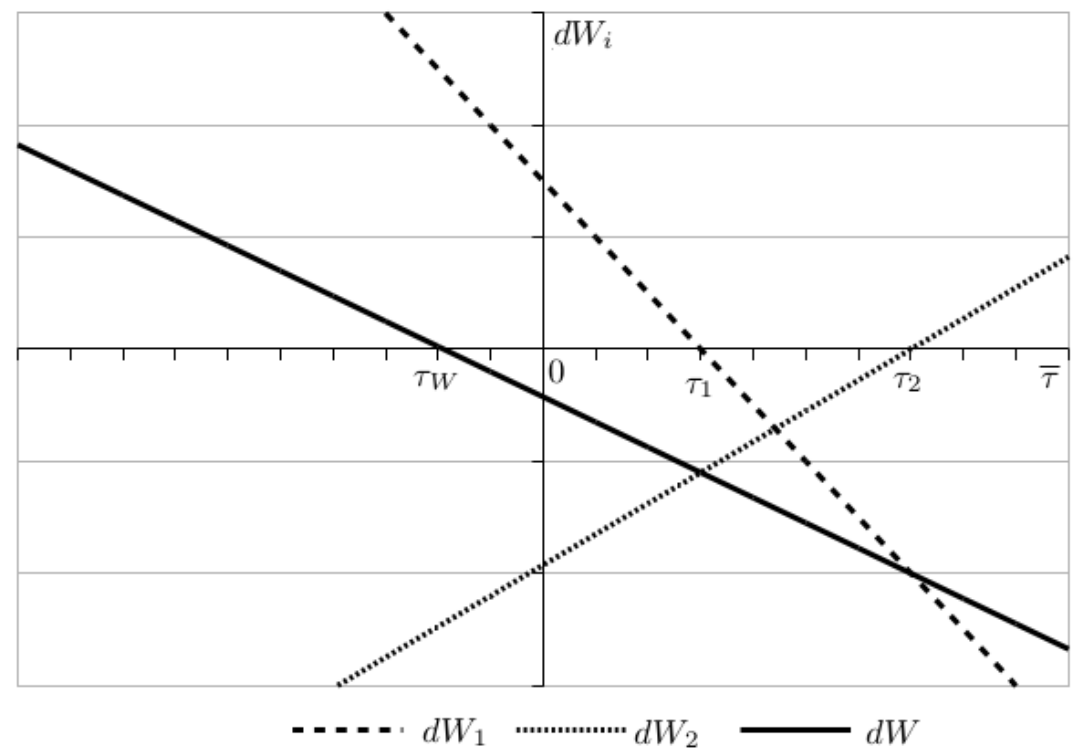

En el punto exacto en que $\omega_{2}$ es igual a $-\omega_{1}$ el SWTRI está indeterminado. Esto se debe a que, dado que los efectos de bienestar se cancelan, no existe arancel unificado que lleve al mismo nivel de bienestar. Gráficamente, la pendiente de $d W$ es cero, con lo que cruza al eje de las abscisas en infinitos puntos. Algebraicamente, el denominador de la ecuación (9) es igual a cero.

$\mathrm{El}$ arancel del bien 2, $\tau_{2}$ tampoco es un límite superior para el SWTRI. Si $\omega_{2}$ se incrementa por encima de $-\omega_{1}$ el SWTRI se vuelve mayor a $\tau_{2}$. En este caso particular $d W>0$, con lo que subir los aranceles en su conjunto aumenta el bienestar. La interpretación es que dado su ponderación fuerte de los hogares más pobres, para el SWTRI el país en el agregado es un productor neto de estos bienes. En realidad el SWTRI no es tanto un arancel en este caso, sino un subsidio a la exportación. 
Figura 5: Un bien importable y otro que se vuelve exportable $\omega_{1}<0<\omega_{2}$

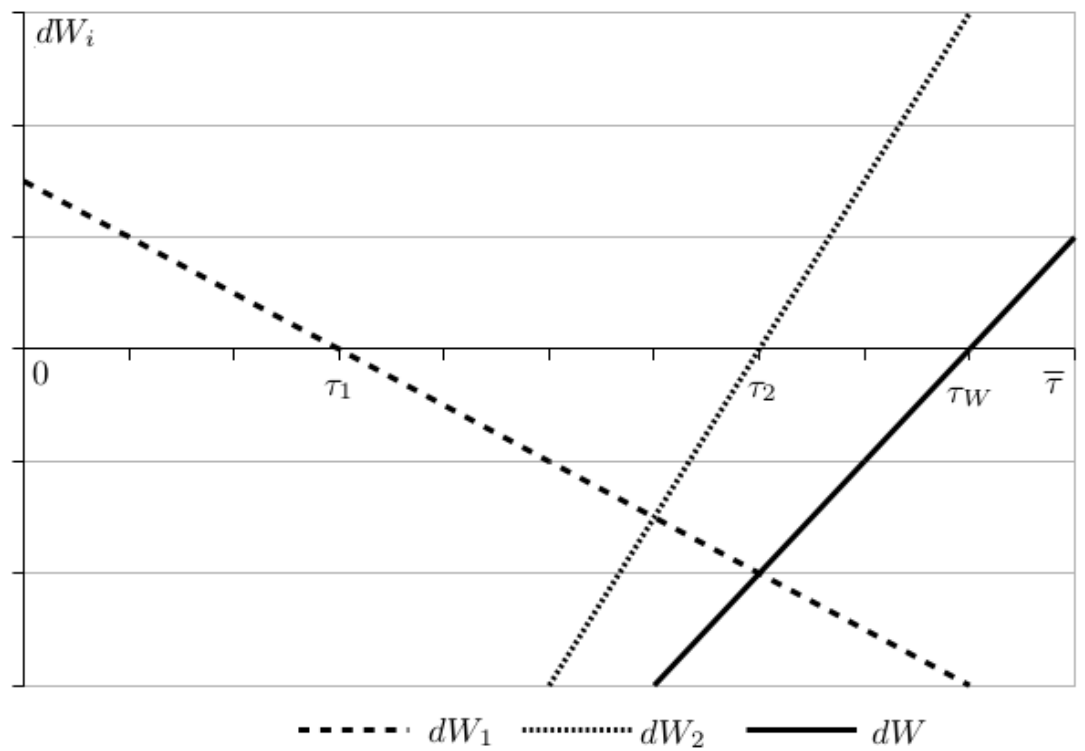

Adelantando resultados mencionamos que los casos con $d W>0$ e incluso $d W=0$ son teóricamente posibles pero no se han encontrado en los análisis empíricos. En todos los casos se observa que tanto en la agregación total de los bienes en $d W$ como en sub-agregaciones como industria y agricultura se mantiene el signo negativo, aún a altos niveles de épsilon.

\section{Implementación empírica}

En la sección anterior se resolvió algebraicamente el SWTRI. Se obtuvo partiendo de la definición una expresión teórica y se desarrolló sobre su funcionamiento teórico. En esta sección se define cómo implementar las fórmulas en base a datos de la realidad. A tal motivo se toma como ejemplo a Burkina Faso.

La ecuación (7) requiere tres variables para ser calculada. En primer lugar $x^{h}$, el ingreso real de cada hogar, el cual se obtiene de encuestas de hogares. También se necesita informacion de $\tau_{i}$, los aranceles, los cuales se obtienen de bases de datos de comercio internacional. Finalmente, y de mayor importancia se necesita estimar $\frac{\partial x^{h}}{\partial \tau_{i}}$, el efecto de cada arancel sobre el ingreso real de cada hogar. A continuación se discutirán los desafíos que plantea cada etapa y como se decidió resolverlos.

\subsection{Especificación del efecto de los aranceles sobre el in- greso real.}

Estimar el impacto de la política comercial sobre el ingreso real es un ejercicio por más complejo. La política comercial afecta al ingreso de las personas a través de varios mecanismos. Una serie de mecanismos posible es la siguiente: 
En primer lugar altera precios relativos de los hogares, al elevar los aranceles el precio interno de los bienes. A su vez, esos efectos afectan las decisiones de producción de las empresas, demandando más o menos trabajo en cada industria, alterando no sólo precios de bienes sino de factores. Pero naturalmente el cambio de precios reconfigura la matriz productiva del país. Esa reconfiguración puede alterar los costos productivos en el corto plazo según la pendiente de los costos marginales de cada industria, y en el largo plazo con el reescalamiento industrial y efectos de aprendizaje. Esas nuevas industrias pueden a su vez incentivar la acumulación de desarrollo humano de los hogares, alterando la dotación factorial y no solo la remuneración factorial que cada hogar enfrenta.

Describir en forma completa la complejidad de los efectos de la polítca comercial sobre el ingreso real de cada hogar está por fuera de los objetivos de este trabajo. Solo se busca generar un marco teóricamente sólido para poder implementar la ecuación (7). Para ello se harán supuestos simplificadores que harán el cálculo además de fácilmente implementable, transparente.

Los supuestos que se emplean son los siguientes. En primer lugar los hogares que producen bienes transables lo hacen en competencia perfecta y no enfrentan ninguna brecha entre precio local y precio internacional que los aranceles y el tipo de cambio, que se mantiene fijo. La misma estructura de competencia se da para los hogares como consumidores, con lo cual nuevamente enfrentan el precio de los bienes que consumen es el precio internacional multiplicado por el tipo de cambio y los aranceles. Alternativas a estos supuestos serían plantear modelos de competencia imperfecta simples, como monopsonio en bienes exportables o monopolio en bienes importables. Modelos de largo plazo podrían ajustar los costos de los hogares en base al reescalamiento de cada firma.

En segundo lugar el mercado de trabajo sigue los supuestos del modelo ricardiano. Esto implica que el trabajo recibe el $100 \%$ de la variación del precio del bien, que a su vez, dado la cláusula de ceteris paribus implícita en el trabajo, se corresponde con la variación del arancel. Otros trabajos complejizan este supuesto estimando los efectos de los aranceles sobre factores en base a series de tiempo. Una solución elegante, de Nicita, Olarreaga y Porto(2014), es emplear el Teorema de Young sobre una función de producción del PBI para calcular el efecto marginal de los precios sobre los salarios a través de su equivalente de derivadas cruzadas, el efecto marginal de los insumos sobre el PBI. También se puede relajar el supuesto de competencia perfecta, introduciendo fricciones al mercado de trabajo como en Artuç, Chauduri, McLaren (2010) y Artuç, Lederman, Porto (2015).

En tercer lugar dejamos a los precios de los no transables fijos. Es decir, el cambio de aranceles no afectará al ingreso de los trabajadores o productores del sector no transable sino a través de la alteración ocurrida en el precio de los transables que consumen. En cambio en los trabajos de Porto $(2005,2006)$ se tiene en cuenta efectos de la política comercial sobre la determinación de los precios de los no transables también.

Finalmente, se toma como supuesto que el comportamiento del estado no cambiará ante cambios de la recaudación por aranceles, simplemente ahorra o desahorra. Una mayor o menor recaudación por no afectará la asistencia social, gasto público, tasas de interés a la que se financia el Estado, etc. Esto es equivalente a suponer que el gasto público se realiza en forma proporcional al ingreso. Estrategias alternativas serían por ejemplo la empleada por Nicita Olarreaga, Porto (2014), quienes realizan un meta-estudio de incidencia distributiva 
del gasto en la región, recopilando resultados obtenidos por otros autores respecto a la incidencia distributiva del gasto y la aplican en el caso particular. La solución más precisa y demandante es realizar un análisis de incidencia del gasto del país sobre la distribución del ingreso como la realizada para Argentina en Gasparini (1998), y aplicar la recaudación en base a la incidencia promedio.

Habiendo explicitado todos nuestros supuestos se está en condiciones de obtener expresiones algebraicas que hagan la implementación directa. Se empieza definiendo a $x^{h}=\frac{y^{h}}{P^{h}}$, donde $y^{h}$ es el ingreso nominal del hogar $h$ y $P^{h}$ su "índice de precios" . El ingreso nominal es la suma de salarios, $w^{h}$, beneficios, $\pi_{i}$, y la proporción de recaudación por aranceles. El índice de precios del hogar se define como el promedio de los precios de los bienes que enfrenta cada hogar, ponderado por su participación presupuestaria. Esto hace que el ingreso real del hogar quede definido como

$$
x^{h}=\frac{y^{h}}{P^{h}}=\frac{w^{h}+\sum_{i} \pi\left(p_{i}\right)}{\prod_{i}\left(p_{i}\right)^{s_{i}^{h}}}
$$

Donde $s_{i}^{h}$ es el cociente entre el gasto del hogar $h$ en el bien $i$ y el consumo total del hogar $h$. Diferenciando, y en base a nuestros supuestos se obtiene

$$
\begin{gathered}
\frac{\partial \ln P^{h}}{\partial \ln \tau_{i}}=s_{i}^{h} \frac{\tau_{i}}{1+\tau_{i}} \\
\frac{\partial \ln y^{h}}{\partial \ln \tau_{i}}=\theta_{w}^{h} \varepsilon_{w p_{i}} \frac{\tau_{i}}{1+\tau_{i}}+\theta_{\pi_{i}} \frac{\tau_{i}}{1+\tau_{i}}
\end{gathered}
$$

Con lo que bajo nuestra especificación de los efectos de los aranceles, el SWTRI queda definido como:

$$
\tau^{W}=\frac{\sum_{h} \sum_{i}\left(x^{h}(\tau)\right)^{1-\epsilon}\left[\theta_{w}^{h} \varepsilon_{w p_{i}}+\theta_{\pi_{i}}^{h}-s_{i}^{h}\right] \frac{\tau_{i}}{1+\tau_{i}}}{\sum_{h} \sum_{i}\left(x^{h}(\tau)\right)^{1-\epsilon}\left[\theta_{w}^{h} \varepsilon_{w p_{i}}+\theta_{\pi_{i}}^{h}-s_{i}^{h}\right] \frac{1}{1+\tau_{i}}}
$$

Donde $\theta_{w}^{h}$ es la proporción que representan los salarios en el ingreso total del hogar $h, \theta_{\pi_{i}}^{h}$ es la proporción que representan los benefcios derivados de la producción del bien $i$ en el ingreso total del hogar $h$ y $\varepsilon_{w p_{i}}$ es la elasticidad de los salarios respecto a las variaciones del precio del bien $i$. Bajo el supuesto ricardiano, el trabajo absorbe todas las variaciones de los precios, con lo cual $\varepsilon_{w p_{i}}=1$.

Las variables $\theta_{w}^{h}, \theta_{\pi_{i}}^{h}$ definidas son directamente estimables de encuestas de hogares. Lo único que se necesita notar es que se definirán a los sectores industriales y agriculturales como transables, y al sector servicios como no transable, sólo los aranceles de los sectores transables tendrán efectos sobre el ingreso real.

\subsection{Datos de encuestas de hogares}

En esta sección se especificará la fuente para obtener datos sobre el gasto e ingreso de los hogares y cuáles fueron las modificaciones que se le hicieron para que puedan ser empleados por el modelo empleado.

La fuente que se emplea es la Encuesta de hogares de Burkina Faso correspondiente al año 2003, o Enquête Burkinaibe sur les conditions de vie des 
ménages de 2003 en su idioma original. La encuesta corresponde al trabajo de Nicita, Olarreaga y Porto (2014).

Los estadísticos descriptivos se muestran en la Tabla 1. La encuesta posee 8500 observaciones, tanto de hogares rurales como urbanos.

La base de datos también incluye información sobre las características de los jefes de hogares. De los 44 años de vida promedio de los jefes de hogar, solo 1,52 fueron de escolarización. Las mujeres estan fuertemente subrepresentadas, ya que no llegan ni al 10\% del total de los jefes. Contando al jefe, cada familia posee 5,57 integrantes.

Finalmente, notamos que Burkina Faso es un país altamente desigual. El índice de Gini calculado sobre la distribución del consumo per cápita arroja un nivel cercano al 0,45, y con respecto al ingreso 0,57.

La conclusión que emerge de estos datos es que Burkina Faso en 2003 era un país cuyos habitantes no poseían un elevado nivel de instrucción promedio, de tamaño familiar elevado y que vivían mayormente en el campo. Esto configuraba una economía de hogares-firma, que vivían de la producción de bienes agriculturales tanto como para auto consumo como para venta. A su vez, la desigualdad, medida tanto como del ingreso como del consumo era elevada. Por ende, una herramienta que podría usar el gobierno de este país es la política comercial: gravando fuertemente a los bienes que producían los hogares menos pudientes, aumentando su precio interno, y desprotegiendo los bienes de los hogares más ricos.

Tabla 1: Estadísticos descriptivos de la Encuesta de Hogares de Burkina Faso

\begin{tabular}{|l|c|}
\hline Año & 2003 \\
\hline Observaciones (hogares) & 8500 \\
\hline Hogares rurales (\%) & 79,2 \\
\hline Ingreso proveniente de autoconsumo (\%) & 36,4 \\
\hline Personas promedio por hogar & 5,57 \\
\hline Edad promedio del jefe de hogar (años) & 44,38 \\
\hline Años promedio de escolarización del jefe hogar (años) & 1,52 \\
\hline Mujeres jefe de hogar (\%) & 8,13 \\
\hline Gini del consumo per cápita familiar & 0,448 \\
\hline Gini del ingreso per cápita familiar & 0,569 \\
\hline
\end{tabular}

La base de datos de Burkina Faso se obtuvo directamente de los autores, con lo que se encuentra semi-procesada. Como mencionan en su trabajo, los ingresos y gastos rurales se encuentran desagregados a 3 dígitos, los gastos en manufacturas y servicios a 1 y los ingresos laborales se encuentran discriminados por sector, a 1 dígito. La clasificación completa se encuentra especificada en las tablas 2 a 5 .

Se debe hacer un comentario final respecto del procesamiento de las participaciones. En el ejemplo teórico teníamos dos bienes importables. En la encuesta de hogares existen tanto bienes importables como exportables. Esto lleva a decidir sobre cómo distinguir entre ellos.

Una opción es comprobar cuáles son los bienes que los hogares son en conjunto productores y consumidores netos. Esto se hace fácilmente, evaluando el signo de cada $\omega_{i}$ cuando $\epsilon=0$. Sin embargo, no se hizo tal cosa debido a que los 
Tabla 2: Clasificación de ingresos monetarios

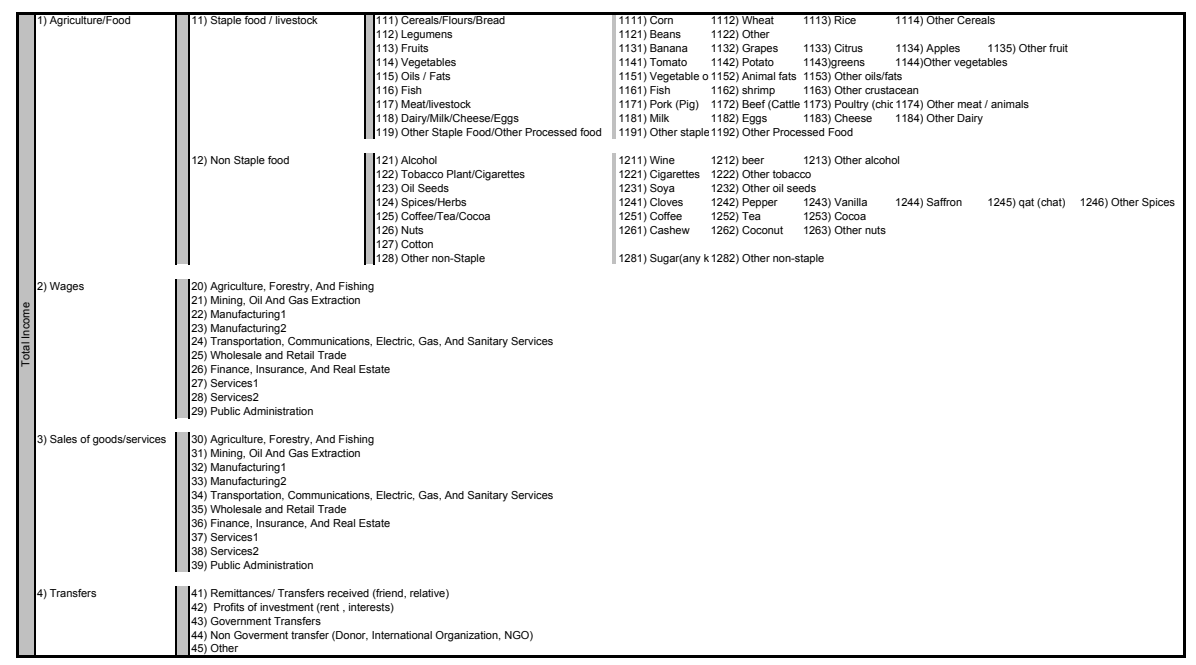

Tabla 3: Clasificación de ingresos no monetarios



datos con los que se cuenta difieren del ejemplo en dos aspectos fundamentales.

En primer lugar, como se posee un relativamente bajo nivel de desagregación, nada garantiza que bienes importables y exportables estén incluidos en la misma categoría. Con lo cual al eliminar la categoría estamos eliminando tanto bienes importables como exportables.

En segundo lugar, algunas categorías poseen $\omega_{i}>0$ pero de ellas no se puede inferir que correspondan a un bien en el cual el país es un exportador neto. Se trata de las categorías de salario, que son para las encuestas de hogares siempre de $\omega_{i}>0$. Ignorar las categorías con $\omega_{i}>0$ llevaría entonces a ignorar a todos los efectos de la política comercial en los salarios.

Por estas dos razones se decidió incluir a todas las categorías en el cálculo del SWTRI.

\subsection{Datos de comercio internacional}

Habiendo obtenido las participaciones de los gastos e ingresos en el ingreso de los hogares, el ingreso real per cápita y la elasticidad precio de los salarios, solo queda por obtener los aranceles. Esta sección se aboca a contar de donde se obtuvieron y cómo se calcularon. 
Tabla 4: Clasificación de gastos

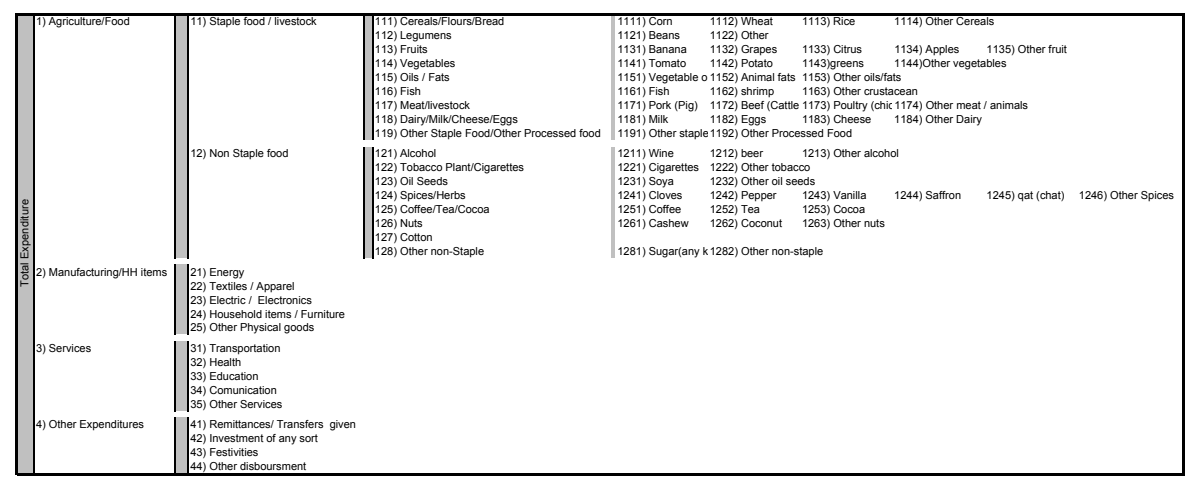

La fuente de los $\tau_{i}$ fue la World Integrated Trade Solutions del Banco Mundial ${ }^{2}$. Dada la desagregación que tenía los datos de encuestas de hogares, una desagregación a 4 dígitos del Sistema Armonizado (HS4 por sus siglas en inglés) fue suficiente. Luego se hizo corresponder cada código arancelario del HS4 a la codificación de gastos e ingresos que llamamos hh4. Como esta correspondencia lleva varias páginas no la publicamos aquí, pero está a disposición del lector bajo pedido al autor.

Dada la naturaleza de los datos, hay en general varias categorías de comercio por cada categoría de ingreso/gasto. Es por esto que se debía realizar un promedio previo para obtener un valor único de arancel para cada categoría de gasto e ingreso, como requiere la teoría. Siendo coherentes con la metodología que se emplea para agregar dentro del sistema armonizado (por ejemplo, de HS6 a HS4), el método de agregación empleado fue el promedio ponderado por importaciones.

Se presentan los estadísticos descriptivos de los datos de aranceles de Burkina Faso en la Tabla ??. Se observa que el rango de aranceles va del 0 al 20\%, con una media de $12,51 \%$ y una mediana de $10 \%$. Esto indica que hay una asimetría a derecha: hay pocos códigos arancelarios que tienen arancel máximo de $20 \%$.

Tabla 5: Estadísticos descriptivos de los aranceles de Burkina Faso

\begin{tabular}{l|cc} 
Estadístico & HS4 & hh4 \\
\hline Mínimo & $00,00 \%$ & $05,00 \%$ \\
Quintil 1 & $05,00 \%$ & $08,89 \%$ \\
Quintil 2 & $10,00 \%$ & $12,50 \%$ \\
Mediana & $10,00 \%$ & $18,76 \%$ \\
Media & $12,51 \%$ & $14,98 \%$ \\
Quintil 3 & $17,51 \%$ & $19,96 \%$ \\
Quintil 4 & $20,00 \%$ & $20,00 \%$ \\
Máximo & $20,00 \%$ & $20,00 \%$ \\
Desvío Estándar & $06,77 \%$ & $05,63 \%$
\end{tabular}

Otro fenómeno que se observa es que la agregación tiende a correr la distribución a la derecha: todos los estadísticos de posición aumentan. Esto se

${ }^{2}$ http://wits.worldbank.org/ 
debe a la naturaleza de la política comercial de Burkina Faso y nuestro patrón de desagregación. Burkina Faso, como muchos países en desarrollo grava más fuertemente a la importación de bienes agriculturales. Para el código 0 del sistema armonizado (Animales y productos animales, y productos vegetales), por ejemplo el arancel promedio es 17,8\%. A su vez, nuestra desagregación es rica en este tipo de bienes: hay desagregación a 3 dígitos mientras que solo a un dígito en manufacturas. Esto genera muchos aranceles altos. Sin embargo, esto no es un inconveniente para el cálculo del SWTRI, ya que estos códigos de alta desagregación también tienen un efecto menor sobre el bienestar, al ser bienes con poca participación promedio en el gasto/ingreso de los hogares. Al tener poca participación promedio el ponderador $\omega_{i}$ de estos códigos será bajo. Con lo que la distribución contra la cual se debe comparar el SWTRI es la orginal, y no el agregado de hh4.

\section{Resultados}

Se muestran los resultados obtenidos del cálculo del SWTRI para Burkina Faso para distintos niveles de $\epsilon$ en la Figura 6. Vale recordar que el intervalo en en cual Burkina Faso estableció aranceles es del 0 al 20\%, siendo el promedio ponderado ligeramente inferior a 10\%. Notamos también que el SWTRI decrece a medida que aumentamos épsilon, de un nivel de aproximadamente $11,4 \%$ a $7,6 \%$, reduciéndose en 3,8 puntos porcentuales o $33 \%$.

Figura 6: El SWTRI para distintos niveles de $\epsilon$ en Burkina Faso



La interpretación que emerge de este gráfico es que Burkina Faso es menos proteccionista de lo que aparenta cuando corregimos por preferencia de desigualdad. Lo que parece evidenciarse es que la política comercial de Burkina Faso es pro-pobre. Estos resultados están de acuerdo a lo que obtienen Nicita, Olarreaga y Porto(2014). 
Para mostrar un gráfico similar al presentado en el ejemplo teórico, se realiza una agregación a dos bienes: agricultura y manufacturas. Para ello se suman todos $\operatorname{los} d W_{i}$ de agricultura por un lado y todos los $d W_{i}$ de manufactura por el otro. Es decir se define

$$
d W_{I}=\frac{\bar{\tau}-\tau_{I}}{1+\tau_{I}} \sum_{i \in I} \sum_{h}\left(x^{h}\right)^{1-\epsilon}\left[\theta_{w}^{h} \varepsilon_{w p_{i}}+\theta_{\pi_{i}}^{h}-s_{i}^{h}\right]
$$

Luego para cada nivel de agregación existirá un "sub-SWTRI" por cada sector $I, \tau_{I}$, que será el arancel aplicado al sector $I$ que mantendría el nivel de bienestar de los hogares constante. Algebraicamente

$$
\tau_{I}=\frac{\sum_{h} \sum_{i \in I}\left(x^{h}\right)^{1-\epsilon}\left[\theta_{w}^{h} \varepsilon_{w p_{i}}+\theta_{\pi_{i}}^{h}-s_{i}^{h}\right] \frac{\tau_{i}}{1+\tau_{i}}}{\sum_{h} \sum_{i \in I}\left(x^{h}\right)^{1-\epsilon}\left[\theta_{w}^{h} \varepsilon_{w p_{i}}+\theta_{\pi_{i}}^{h}-s_{i}^{h}\right] \frac{1}{1+\tau_{i}}}
$$

Estos $d W_{I}$ se encuentran calculados para $\epsilon=0$ y $\epsilon=2$ en las Figuras 7 y 8.

Figura 7: Diferenciales de bienestar en Burkina Faso con $\epsilon=0$




Figura 8: Diferenciales de bienestar en Burkina Faso con $\epsilon=2$

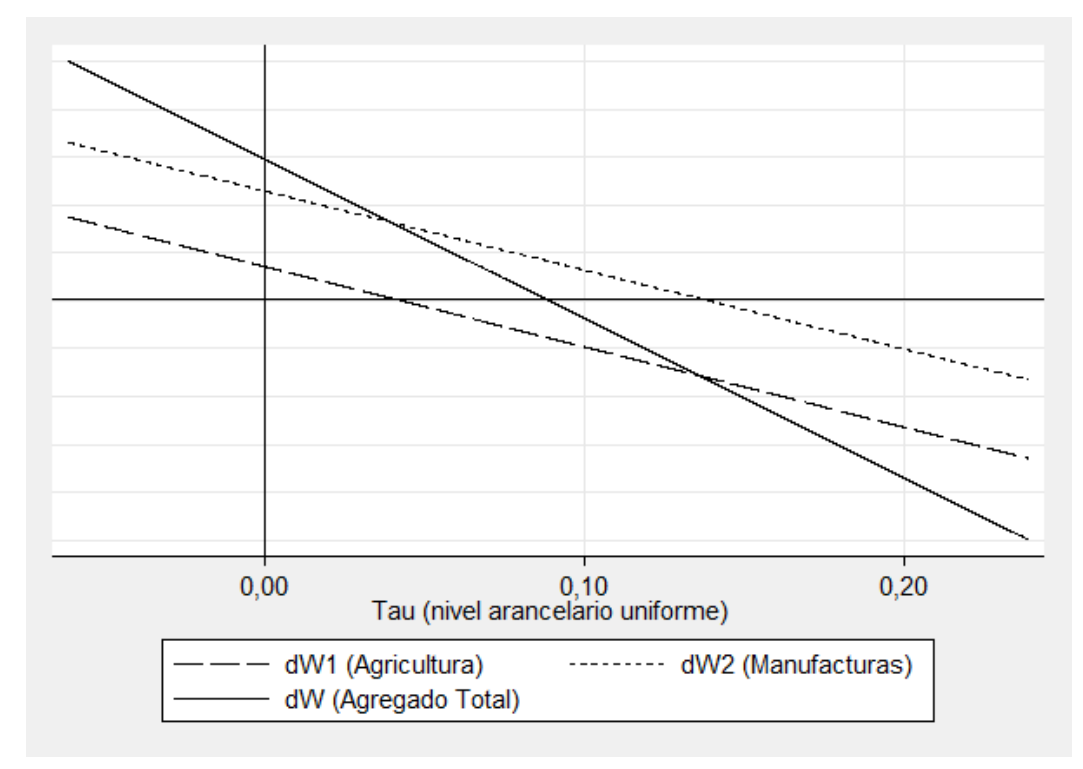

En primer lugar en ambos gráficos que las pendientes de los diferenciales tienen el signo deseado, negativo. En segundo lugar las figuras muestran que el sub-SWTRI de agricultura es menor que el de manufacturas. Esto parecería ser contradictorio con lo mostrado en la sección 3.3, en la que se dijo que el arancel promedio para agricultura es mayor que el arancel promedio en manufacturas. Sin embargo, estos sub-SWTRIs son promedios de aranceles ponderados por efecto sobre el bienestar social, y como se mostró en el ejemplo de dos bienes en la sección 2.4 pueden tomar cualquier valor real. Lo que este cambio muestra es que en agricultura, Burkina Faso no es tan proteccionista como parece, cobrándole un arancel más alto a los bienes con efectos negativos menores (o positivos, ya que para algunos bienes agriculturales Burkina Faso es productor neto) y más bajo a los bienes con fuertes efectos de bienestar muy negativos.

La naturaleza particular de agricultura se vuelve más pronunciada a medida que aumenta $\epsilon$. La reducción en el SWTRI se debe a un corrimiento fuerte hacia la izquierda del $\tau_{1}$, el sub-swtri correspondiente a agricultura, incluso por debajo del arancel mínimo en ese sector. Este comportamiento es el descripto en la Figura 4 en la sección 2.4. Como en aquel caso, el fenómeno se debe al proteccionismo en los bienes en los cuales los hogares más pobres son productores netos. A medida que aumenta $\epsilon$ y se pondera de manera más fuerte a los hogares más pobres, BFA emerge como productor neto de muchos bienes. De los 42 bienes agriculturales, con $\epsilon=0$ Burkina Faso es productor neto en 9, mientras que con $\epsilon=2$ es productor neto en 21 de los 42 bienes.

La conclusión que se deriva de esta sección es que Burkina Faso no es tan proteccionista en términos distributivos. Y a medida que aumenta la ponderación de los hogares más pobres, se vuelve aún menos proteccionista. El mecanismo redistributivo de la política comercial se encuentra en la agricultura. Burkina Faso cobra aranceles altos en los bienes importables que compiten con la producción de los hogares rurales más pobres. 


\section{Conclusiones}

La literatura de protección comercial se ha enfocado en describir el grado de proteccionismo de un país en base a los efectos que aranceles y trabas cuantitativas tienen sobre la eficiencia económica. En contraste con esta postura, este trabajo se centró en desarrollar un índice que tome en cuenta los efectos distributivos de la política comercial.

En base a una función de bienestar Atkinson (1970) se desarrolló un índice de que permite calcular el grado de proteccionismo de un país en base a los efectos de los aranceles sobre el ingreso real, ponderando a cada hogar en función del parámetro $\epsilon$ que resume las preferencias por la igualdad del analista. En línea con la nomenclatura de la literatura se lo llamó SWTRI, Social Welfare Trade Restrictiveness Index, índice de protección comercial basado en el bienestar social.

Este índice puede ser entendido como un promedio ponderado de los efectos de bienestar de los aranceles de un país. Si los aranceles altos son los que más afectan al bienestar social, luego el índice será mayor al promedio. En cambio, si el país establece aranceles menores a los bienes con un fuerte impacto en el bienestar social, el índice será menor.

El índice no está restringido al rango de los aranceles. Un índice menor al mínimo de los aranceles se debe a que el país está cobrando aranceles altos a bienes en los cuales el país es productor neto. El índice puede ser incluso negativo. La interpretación de este caso particular es que la política comercial es tan redistributiva, que para generar el mismo nivel de bienestar con una política arancelaria uniforme, debería haber un subsidio generalizado a las importaciones.

Al implementar el modelo para Burkina Faso se encuentra que el país en efecto utiliza su política comercial con fines redistributivos. Para un nivel de preferencia de igualdad nula, $\operatorname{con} \epsilon=0$ el índice no difiere de manera significativa de un promedio ponderado por importaciones. Sin embargo, a medida que aumenta la preferencia por igualdad, el índice empieza a caer. El mecanismo por el cual el índice cae es la redistribución que tiene lugar dentro del sector agricultural: Burkina Faso cobra aranceles altos a los bienes producidos por los hogares rurales más pobres. 


\section{Bibliografía}

Anderson, James y Peter Neary (1992), "Trade reforms with quotas, partial rent retention and tariffs", Econometrica, 60(1), 57-76.

Anderson, James y Peter Neary (1994), "Measuring the restrictiveness of trade policy", World Bank Economic Review, 8, 151-169.

Anderson, James and Peter Neary (1996), "A new approach to evaluating trade policy", Review of Economic Studies, 63(1), 107-125.

Anderson, James and Peter Neary (2003), "The Mercantilist index of trade policy", International Economic Review 44(2), 627-649.

Anderson, James and Peter Neary (2004), "Welfare versus market access: the implications of tariff structure for tariff reform", NBER working paper \# 10730.

Antràs, Pol, Alonso de Gortari, and Oleg Itskhoki (2015). "Inequality, Costly Redistribution and Welfare in an Open Economy". Working paper disponible en http://scholar.harvard.edu/antras/publications/inequality-costly-redistributionand-welfare-open-economy

Artuç, Erhan, Shubham Chaudhuri, y John McLaren (2010). "Trade Shocks and Labor Adjustment: A Structural Empirical Approach." American Economic Review, 100(3): 1008-45.

Artuc, Ethan , Lederman, Daniel Porto, Guido (2015). A mapping of labor mobility costs in the developing world. Journal of International Economics, $95(1)$.

Atkin, David y Donaldson, Dave (2015). "Who's Getting Globalized? The Size and Implications of Intra-national Trade Costs," NBER Working Papers 21439, National Bureau of Economic Research, Inc.

Atkison, B. Anthony. (1970) "On the Measurement of Inequality" Journal of Economic Theory. 2 244-263

Fajgelbaum, Pablo D. y Khandelwal, Amit F (2016). "Measuring the Unequal Gains from Trade", Quarterly Journal of Economics.

Galle, Simon Rodríguez-Clare, Andrés Yi, Moises. (2015) "Slicing the Pie: Quantifying the Aggregate and Distributional Consequences of Trade" Borrador disponible en http://eml.berkeley.edu/ arodeml/ .

Gasparini, Leonardo C. (1998) "Incidencia distributiva del sistema impositivo argentino". Cap 12 en "Reforma Tributaria en la Argentina". FIEL

Kee, Hiau Looi; Nicita, Alessandro y Olarreaga, Marcelo, (2006). "Estimating trade restrictiveness indices" Policy Research Working Paper Series 3840, The World Bank. 
Nicita, Alessandro; Olarreaga, Marcelo y Porto, Guido (2014). "Pro-poor trade policy in Sub-Saharan Africa," Journal of International Economics, Elsevier, vol. 92(2), páginas 252-265.

Porto, Guido G., (2005). "Informal export barriers and poverty," Journal of International Economics, Elsevier, vol. 66(2), páginas 447-470, Julio.

Porto, Guido G., (2006). "Using survey data to assess the distributional effects of trade policy," Journal of International Economics, Elsevier, vol. 70(1), páginas 140-160, Septiembre. 